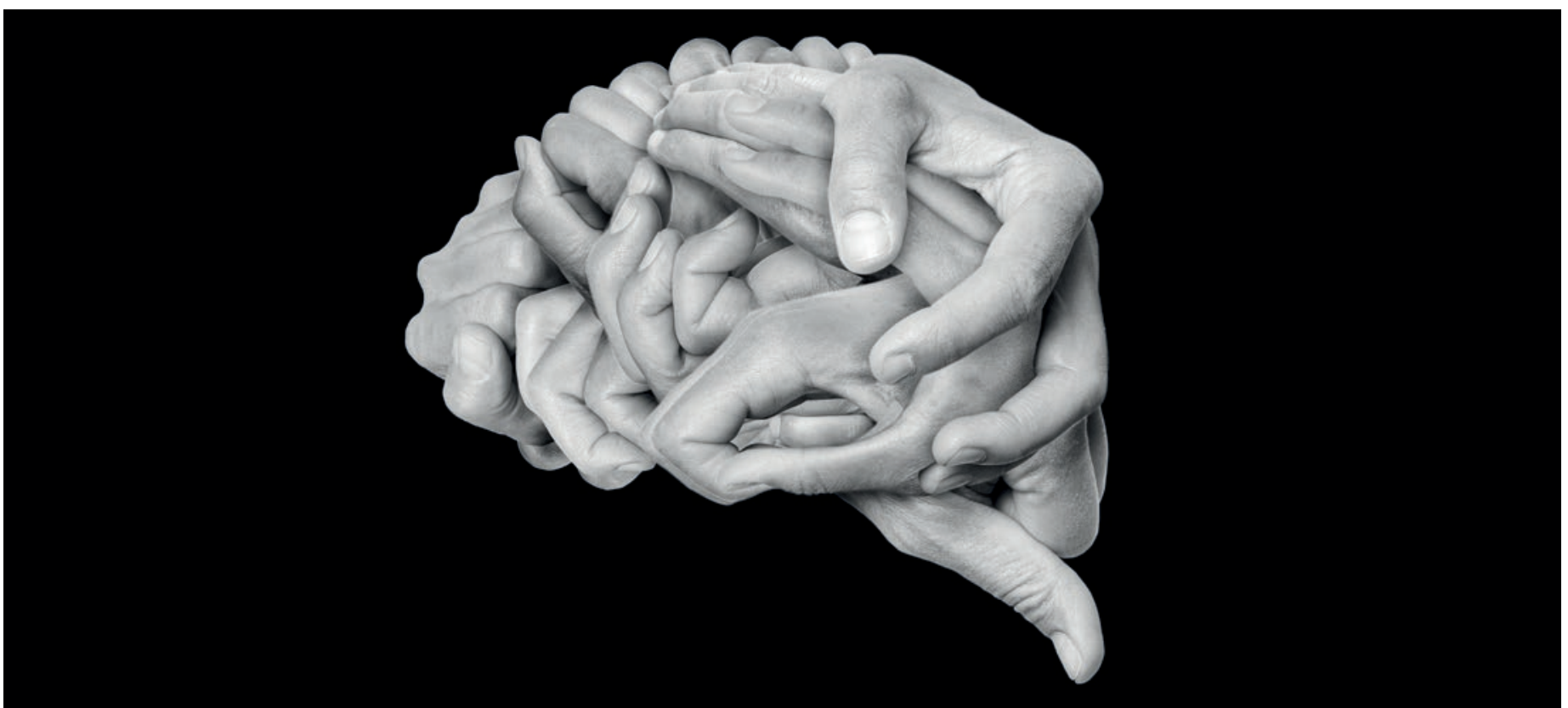

Les Women in Neurology se mettent en réseau pour promouvoir les carrières féminines

\title{
Women in Neurology: Promouvoir l'égalité des chances
}

\section{Barbara Tettenborn ${ }^{a}$, Andrea Humm ${ }^{b}$}

a Prof. Dr méd., présidente de "Women in Neurology», Hôpital cantonal de Saint-Gall, Saint-Gall;

b PD Dr méd., assesseure au sein du Comité de la Société Suisse de Neurologie (SSN), HFR Fribourg - Hôpital cantonal, Fribourg

Les «Women in Neurology», WIN en abrégé, font partie de la Société Suisse de Neurologie. Elles veulent promouvoir les opportunités de carrière pour les femmes médecins et s'appuient sur les programmes de mentorat et le réseautage.

Depuis plusieurs années, ce sont avant tout des femmes qui étudient la médecine et suivent la formation postgraduée pour devenir spécialistes en neurologie. Pourtant, dans le domaine de la neurologie, elles sont encore en minorité et largement sous-représentées parmi les médecins-cadres, les médecins-adjoints resp. -adjointes et les médecins-chefs resp. -cheffes.

Ainsi, une enquête récemment menée auprès des établissements de formation postgraduée en neurologie de catégorie A a révélé que $70 \%$ des médecins assistants et assistantes sont des femmes. Parmi les chefs et cheffes de clinique, la proportion de femmes diminue à 50\% et parmi les médecins-chefs resp. -cheffes et les médecins-adjoints resp. -adjointes, il y a moins de $20 \%$ de femmes.
Pour cette raison, un ensemble de femmes neurologues expérimentées, engagées dans la Société Suisse de Neurologie (SSN) et issues de toute la Suisse, a fondé le 12 février 2020 à Olten un groupe spécialement dédié aux femmes médecins assistantes et spécialistes en neurologie: Women in Neurology, en abrégé WIN.

\section{Programme de mentorat}

En tant qu'association de la SSN, WIN représente les intérêts de toutes les femmes médecins assistantes et spécialistes en neurologie. Son objectif majeur est de soutenir et d'améliorer l'égalité des chances et les possibilités de carrière des femmes dans le domaine de la neurologie. L'association souhaite permettre aux 


\section{Le Comité de Women in Neurology} (WIN)

Pr Dr méd. Barbara Tettenborn, présidente

PD Dr méd. Susanne Renaud, vice-présidente

Pr Dr méd. Margitta Seeck, Academic Development

PD Dr méd. Mira Katan Kahles, Coaching/Mentoring

Pr Dr méd. Susanne Wegener, Coaching/Mentoring

PD Dr méd. Andrea Humm, représentation SSN

Dr méd. Karin Ackermann, représentation SSNC

PD Dr méd. Marie-Luise Mono, représentation SCS

Pr Dr méd. Caroline Pot Kreis, représentation Société suisse de la SEP

méd. pract. Noëlle Mercier, représentation Ligue Suisse contre I’Epilepsie

femmes médecins assistantes et spécialistes d'entrer en contact avec des collègues de toute la Suisse et d'échanger expériences et idées à un stade précoce de leur carrière.

WIN a la vocation d'être un interlocuteur pour toute question de carrière. Un programme de mentorat est notamment en cours d'élaboration, son but étant de permettre un échange personnel de connaissances et d'expériences entre les femmes neurologues expérimentées occupant des positions dirigeantes et les jeunes femmes cheffes de clinique et médecins assistantes.

Société Suisse de Neurologie (SSN)

c/o IMK Institut pour la

Médecine et la Communi-

cation SA

Münsterberg 1

CH-4001 Bâle

swissneuro[at]imk.ch

www.swissneuro.ch

\section{Contact avec d'autres organisations}

Le contact avec d'autres organisations et instances du domaine de la neurologie et des neurosciences cliniques est tout aussi important que l'échange interne entre les membres de WIN. Un membre du Comité de la SSN de sexe féminin est à chaque fois aussi membre du Comité de WIN afin de garantir un lien étroit entre le Comité de la SSN et l'association. Par ailleurs, dès lors que cela est possible, un membre du Comité de WIN doit être représenté au sein du Comité de la Société Suisse de Neurophysiologie Clinique (SSNC), de la Société Cérébrovasculaire Suisse (SCS), de la Société suisse de la sclérose en plaques et de la Ligue Suisse contre l'Epilepsie. Par la suite, WIN envisage de s'engager dans le réseau de la Swiss Federation of Clinical Neuro-Societies (SFCNS), qui compte 14 sociétés de discipline issues du domaine des neurosciences cliniques.

L'objectif à moyen terme est de faire entendre une voix unifiée au-delà des frontières des différentes neurodisciplines. Lors des réunions annuelles de la SSN, WIN organise à chaque fois un forum afin de discuter de questions importantes et de se positionner par rapport aux défis futurs. La présentation de WIN et de ses activités sur le site internet de la SSN est en cours d'élaboration.

\section{Crédits image symbolique}

Angelo Cordeschi|Dreamstime.com

\section{L'essentiel en bref}

- $70 \%$ des médecins assistants et assistantes sont des femmes. Parmi les médecins-chefs et les médecins-adjoints, on compte moins de $20 \%$ de femmes.

- WIN a la vocation d'être un interlocuteur pour toutes les questions de carrière.

- Grâce à un programme de mentorat et d'une bonne mise en réseau des sociétés professionnelles, les femmes souhaitant faire carrière en tant que neurologues peuvent être soutenues durant leur parcours. 